\title{
EL USO DE COMPUTADORAS EN EL ANÁLISIS DE HISTORIAS VITALES *
}

\author{
JORGE BALÁN, HARLEY L. BROWNING, ELIZABETH JELÍN Y LEE LITZLER ${ }^{1}$ \\ Centro de Estudios de Población, Universidad de Texas
}

\begin{abstract}
Con seguridad se puede decir que los registros de vida personal, tan completos como sea posible, constituyen el tipo perfecto de material sociológico, y que si las ciencias sociales tienen que emplear cualquier otro tipo de material obedece sólo a la dificultad práctica de obtener al momento un número suficiente de tales registros para cubrir la totalidad de los problemas sociológicos y a la enorme cantidad de trabajo que demanda el análisis adecuado de todo el material personal necesario para caracterizar la vida de un grupo social.
\end{abstract}

W. I. Thomas y Florian Znantecki, The Polish Peasant in Europe and America.

Hace ya CASI medio siglo que Thomas y Znaniecki hicieron su llamamiento en favor de un mayor uso de las historias vitales, y cualquier encuesta entre las publicaciones actuales más sobresalientes en las ciencias sociales mostraría que en general su consejo ha pasado desapercibido. Es verdad que la mayoría de los científicos sociales no negarían el valor de las historias vitales en muchos tipos de investigación, sobre todo la relacionada con el ciclo vital. Es innegable que las historias vitales ofrecen riqueza y sentido concreto de detalle y, sobre todo, la oportunidad de examinar la secuencia de comportamiento dentro del más amplio contexto de la vida de una persona. En este sentido, están muy por encima de la mayoría de las encuestas sociales llevadas a cabo en nuestro tiempo. ¿Por qué, entonces, no han logrado mucha aceptación en las ciencias sociales?

Aparte de que son muy costosas cuando se llevan a cabo en gran escala, las historias vitales, como Thomas y Znaniecki lo reconocen,

* Este trabajo aparecerá en inglés en Behavioral Science bajo el título "A Computerized Approach to the Processing and Analysis of Life Histories Obtained in Sample Surveys".

1 Esta investigación deriva de un proyecto patrocinado conjuntamente por el Centro de Investigaciones Económicas de la Facultad de Economía de la Universidad de Nuevo León y el Population Research Center del Departamento de Sociología de la Universidad de Texas en Austin. En ambos centros la investigación ha sido facilitada en parte por donativos de la Fundación Ford. La dirección del proyecto está a cargo de Jorge Balán, Elizabeth Jelín y Harley L. Browning. La traducción al español es de Adalberto García Rocha, de El Colegio de México. 
presentan muchos problemas técnicos en su recolección, proceso y análisis, y más que otra cosa han sido esos problemas, inherentes al manejo de las historias, lo que ha restringido su uso.

Desde el principio no ha quedado muy claro cuál debe ser el alcance de las historias vitales. Ni siquiera Thomas y Znaniecki tuvieron la intención de que éstas fueran "lo más completas posible". Puede verse que su estudio de 300 páginas sobre el inmigrante polaco Wladek fue selectivo en relación con los eventos de la vida de este hombre pertinentes a su asimilación a los Estados Unidos. El problema de la representatividad tampoco ha sido resuelto satisfactoriamente. En consecuencia, algunas historias vitales "completas" sólo han aparecido como suplementos "ilustrativos" en estudios basados en otro tipo de datos; fue éste, en esencia, el procedimiento empleado por Thomas y Znaniecki, y la tradición ha sido mantenida. ${ }^{2}$ En tales situaciones, o bien se pide al individuo que escriba su propia autobiografía de acuerdo con esquemas generales proporcionados por el investigador o se la toma por medio de largas e intensas entrevistas con una grabadora. Oscar Lewis ha aplicado extensamente esta última técnica en sus trabajos recientes (Lewis, 1961, 1966).* La presentación de unas cuantas historias de vida con registros detallados, aun cuando proporcione al lector mejor comprensión de las fuentes sicológicas y sociales del comportamiento individual y de los grupos a que pertenece, deja problemas de interpretación que nunca han sido resueltos de manera satisfactoria. En verdad, algunos de los que han trabajado con estas historias simplemente rehuyen todo esfuerzo analítico a nivel impresionista o sistemático.

Es lamentable que las historias vitales muy pronto fueron identificadas con presentaciones "completas" carentes de análisis sistemático (Dollard, 1935). Se ha desarrollado en la sociología la idea de distinguir las historias vitales de los estudios estadísticos, de suerte que el investigador prospectivo ha sido forzado a creer que tiene que elegir entre trabajar con historias de vida o con datos que pueda manipular estadísticamente. Es así como las historias de vida han llegado a ser consideradas cada vez más como información "cualitativa" y como datos "blandos" (Young, 1966). No obstante, aun en los estudios de mayor orientación estadística, la necesidad de contar con información biográfica o longitudinal pronto se ha hecho aparente, particularmente en el campo de los estudios de fecundidad, migración y fuerza de trabajo. En estas áreas, el enfoque vía historias de vida es modificado para obtener historias "parciales", es decir, la información se obtiene sólo para cierto aspecto de interés, y algunas veces sólo para una parte del período vital. La estrategia consiste en obtener una secuencia completa de información para el aspecto que interese acerca de la vida del individuo. En este tipo de estudio no hay problema especial de representatividad ya que por regla general. las muestras son grandes, pero hay

2 Por ejemplo, un estudio estadístico en gran escala recientemente publicado sobre Venezuela (Bonilla y Silva Michelena, 1967) incluye una larga historia vital, "Case Analysis of a Revolutionary", por Walter H. Slote, sicoanalista. La historia vital fue incluida en este volumen para demostrar "cómo el juego completo de problemas nacionales presiona la vida de un individuo".

* Véanse las referencias al final. 
una clara restricción en el análisis debido al hecho de que la información de las historias vitales se recoge sólo en relación con un aspecto de la vida del individuo, y por ello no puede establecerse ninguna relación entre dicho aspecto y otras variables. ${ }^{3}$

En este trabajo deseamos fomentar el uso de los datos de las historias vitales mostrando cómo hemos intentado salvar algunos de los problemas técnicos que por mucho tiempo han obstaculizado este tipo de investigación. Las técnicas que presentamos fueron desarrolladas en el transcurso de una encuesta de 1640 hombres entre los 21 y los 60 años de edad en la ciudad de Monterrey. ${ }^{4}$ El propósito principal de la investigación fue determinar la importancia de la movilidad residencial y ocupacional en una ciudad de gran tamaño y de rápido crecimiento en un país en desarrollo. Nuestro enfoque está ubicado en un punto intermedio entre las historias vitales completas, por una parte, y las historias parciales, por la otra. La técnica de obtener y registrar información de historias vitales impone restricciones sobre los tipos de información recogida porque solicita respuesta sólo para ciertos aspectos seleccionados, y, más aún, el entrevistado debe seguir un esquema fijo. Por otra parte, nuestro enfoque difiere de otros estudios parciales porque incluye más de un aspecto. Esto ofrece, en nuestra opinión, ciertas ventajas no sólo en términos de análisis sino en cuanto a confiabilidad de la información obtenida. Este artículo presenta las técnicas de codificación y proceso que fueron desarrolladas haciendo uso de las posibilidades abiertas por las computadoras de alta capacidad. En las pocas ocasiones pasadas en que se ha obtenido gran número de historias vitales, el análisis ha sido siempre inferior a las posibilidades porque no existía manera factible de digerir la gran masa de información (Glass, 1954). La computadora, por primera vez, ha hecho posible analizar sistemáticamente grandes números de historias vitales.

\section{Procedimiento Para la obtención de historias vitales}

La información de historias vitales fue recogida como parte de un cuestionario administrado a todos los entrevistados, en el cual se incluyeron cerca de 200 preguntas (cerradas y abiertas), además de la historia vital. Las entrevistas promediaron casi una hora y media aunque esta cifra estuvo sujeta a amplia variación en función principalmente de la edad del entrevistado y del número de cambios de residencia, trabajo, etc., que había efectuado.

El cuestionario empezó con una serie de preguntas acerca del tra-

3 La mayoria de las encuestas de fecundidad femenina recogen un registro completo de todos los embarazos y muchas también la historia del uso de contraceptivos y otros medios de control de la natalidad (Freedman et al., 1959). Sobre la migración, el estudio reciente más importante fue llevado a cabo por la Oficina del Censo de los Estados Unidos en una historia residencial suplementaria al Current Papulation Survey (Taeuber et al., 1961). Una lista de estudios basados en historias ocupacionales aparece en Wilensky (1960, pp. 553-554).

4 Para una descripción detallada del estudio, véase Jorge Balán, Harley L. Browning y Elizabeth Jelín (compiladores), Movilidad social, migración y fecundidad en Monterrey metropolitano. Monterrey; Centro de Investigaciones Económicas, Facultad de Economía, Universidad de Nuevo León, 1967. 
bajo actual del entrevistado. Luego sigue la cédula para registrar sistemáticamente los datos de historia vital referentes a migración, educación, estado civil y formación de la familia, salud y ocupación. El formato de la cédula de historia vital (véase el anexo 1, p. 441) fue el mismo para todos los entrevistados, sin importar su edad. Las áreas de investigación tienen cada una su columna asignada y cada año posible ( 60 en total) tiene asignado un renglón. El hecho de que apenas unas cuantas historias ocuparon los 60 renglones resultó en que quedó mucho espacio en blanco, pero también aseguró que cada año fuese tomado en cuenta, en vista de que no se permitieron renglones vacíos después de que se había iniciado una actividad (representada por una columna). Esto permitió también la inspección instantánea de las características del entrevistado en cualquier año.

Los enumeradores (en este caso estudiantes varones de la Facultad de Economía de la Universidad de Nuevo León) fueron adiestrados en la administración de la parte de historia vital del cuestionario. Asimismo, fueron estrechamente vigilados para que se pudiera calificar rápidamente la calidad de las entrevistas. Se requirió supervisión estrecha en vista de que las instrucciones dadas a los encuestadores permitían alto grado de discreción durante la entrevista; por esta razón la relación de supervisores a encuestadores fue de $1: 6$.

Las instrucciones generales fueron tomar un aspecto de la historia vital como "foco", seguir la secuencia de sucesos en este aspecto y luego relacionar cada cambio en él con las secuencias en otros aspectos. El aspecto seleccionado como punto de referencia cambió según la edad y la historia particular del encuestado. Generalmente, después de preguntar el año y el lugar de nacimiento, la educación se tomó como "foco" y se relacionó con los cambios de residencia. Luego, el "foco" se cambió a ocupación, o estado civil y familiar, y se siguió con migración para relacionar ésta con los dos aspectos anteriores.

Hubo muchas variaciones en el procedimiento, según la persona, por dos razones. La primera, que hubo diferencias objetivas en las mismas historias vitales. Por ejemplo, la selección de área "focal" fue muy diferente para un hombre de 30 años que había estado en la escuela hasta los 24 años y que nunca había migrado, frente a otro hombre de 60 años con sólo dos de escuela y muchos cambios de residencia. La segunda, que la memoria del entrevistado fue mejor en unos aspectos que en otros. Algunos hombres recordaron los cambios más fácilmente en términos de su historia familiar mientras que otros prefirieron centrar sus recuerdos en relación con su historia ocupacional o con cambios de residencia.

Para que el lector pueda tener idea más concreta de la manera en que se obtuvieron las historias vitales, presentamos a continuación un ejemplo abreviado del procedimiento seguido por el encuestador $\mathbf{y}$ la secuencia de sus preguntas.

Pregunta: ¿Cuántos años tiene usted? ¿Esto es, en qué año nació usted? (Respuesta.)

$P .:$ ¿Dónde nació usted? ¿Dónde vivían sus padres cuando usted nació? $(R$. 
$P$.: ¿Ha asistido usted alguna vez a la escuela? (R.)

$P .: \quad$ Cuándo empezó usted a ir a la escuela? (R.)

$P$.: ¿Vivía usted todavía en el mismo lugar (de nacimiento) cuando empezó a ir a la escuela? (R.) [Si el encuestado se había mudado, se le solicitaron fechas y lugares. Se le preguntó entonces, año por año, si era estudiante, qué era lo que estudiaba, y los resultados - año de estudios, años repetidos, deserción de la escuela- hasta obtener el registro completo de su historia educativa. En cada entrada se le preguntó si vivía aún en el mismo lugar y, si la respuesta era negativa, se le solicitó la información sobre los cambios de residencia y las fechas correspondientes.]

$P$.: Cuando dejó usted la escuela, ¿estaba trabajando? $(R$.

$P .:$ ¿Trabajó alguna vez antes de dejar la escuela? $(R$.

$P$.: ¿Cuándo obtuvo su primer trabajo? ¿Dónde vivía en esa ocasión? ¿Qué estudiaba entonces? $(R$.)

$P .:$ ¿En qué consistía su trabajo? ¿Qué era lo que hacía? (R.)

$P .:$ ¿Cuáles eran sus tareas en ese trabajo? (R.)

$P$.: Trabajaba por su cuenta, era obrero, era empleado o era patrón? (R.)

$P .:$ ¿Tenía usted a su cargo a otras personas? Si es así, ¿cuántas) $(R$.

$P$.: ¿En qué tipo de negocio o empresa trabajaba usted? ¿Qué fabricaban o vendían? ( $R$.)

$P .:$ ¿Cuántas personas trabajaban allí? ( $R$.

$P .:$ ¿Tenía usted algún familiar que trabajaba en la misma empresa o negocio? (R.)

$P .:$ ¿Recuerda usted aproximadamente cuánto ganaba en ese empleo? (R.) [Si la empresa era agrícola, o de alguna actividad relacionada y el entrevistado era el propietario de la tierra, se le preguntó por el tamaño y el tipo de terreno que poseía. A medida que avanzaba la entrevista, el encuestador continuaba su interrogatorio sobre cualquier cambio en ocupación, año por año; por cada cambio registrado, el encuestador introducía preguntas relacionadas con la migración. Al llegar a cierto punto de la historia vital, usualmente a los 25 años de edad del entrevistado, se hizo la siguiente pregunta:]

$P$.: Hasta ese momento, ¿era usted soltero o ya se había casado o "unido"? (R.) [Si la respuesta fue afirmativa, se preguntó la fecha de la unión y la duración del noviazgo. De este punto en adelante, el encuestador continuaba la entrevista relacionando cambios ocupacionales con nacimientos de niños, fallecimientos, terminación de uniones conyugales y cambios de residencia. Si el entrevistado no había facilitado voluntariamente la información, en varios momentos de la historia vital se le preguntó:]

$P .:$ Durante todo este tiempo, ¿padeció usted alguna enfermedad que le impidiera trabajar por algún tiempo o que fuera muy costosa? ¿Qué enfermedad? (R.)

$P$.: ¿Cuándo la padeció? ¿Dónde vivía entonces? ¿En qué trabajaba? (R.)

$P .:$ ¿Padecieron su esposa o sus niños alguna enfermedad grave? ¿Cuál o cuáles? (R.)

$P .:$ ¿Cuándo las padecieron? $(R$. 


\section{Evaluación}

Los problemas de confiabilidad en las respuestas tienen especial significado en la evaluación de las historias vitales. La falsificación deliberada es, por supuesto, problema común a todas las encuestas y se tomaron para esto las precauciones usuales. Debe hacerse hincapié en que no se tenía la intención de penetrar en aspectos sensibles de la vida del entrevistado. Los puntos tocados en la historia vital se referían sólo al comportamiento de la persona (cambios de residencia, empleos, educación) más que a sus actitudes. Somos de la idea de que la falsificación deliberada es menor problema en las encuestas de historias vitales que en encuestas de otro tipo, ya que es claramente más fácil inventar un solo punto de información que una historia de vida completa aparte de que pocas personas tienen el talento para inventar durante una entrevista historias vitales creíbles y consistentes.

El problema de los errores derivados de la dificultad para recordar es más grave y se amplifica naturalmente cuando se pregunta a las personas por acontecimientos que ocurrieron hace muchos años. Sin embargo, las preguntas hechas a encuestados acerca de su niñez son las más fácilmente recordadas, como lugar de nacimiento, número de años completos cursados en la escuela o primer empleo. No obstante, la falsificación no intencional de porciones de la historia vital es fuente importante de error, y es resultado de una o más de las siguientes contingencias: 1) omisión de cambios de staius; 2) ordenación incorrecta de los cambios de status; y 3) asignación incorrecta de las fechas de los cambios de status. Hemos intentado reducir al mínimo estos errores mediante los siguientes procedimientos:

1. Registro sistemático. El formato de la cédula de historias vitales fue diseñado para obligar al encuestador a no omitir ningún año en cada aspecto investigado. Esto también le permitió descubrir inmediatamente los vacíos (años no registrados) que necesitaban ser llenados. En muchos casos este procedimiento ayudó además a establecer buena relación con el entrevistado. El completar la historia vital se convertía así en auténtica empresa conjunta con el encuestador y el encuestado envueltos en un diálogo continuo dirigido a ese propósito.

2. Ayuda para recordar. La probabilidad de que un individuo sea capaz de recordar distintos acontecimientos es mayor si lo puede hacer como parte de una secuencia continua en vez de pedírsele que recuerde algún suceso aislado. Sostenemos que este procedimiento es particularmente útil al trabajar con entrevistados cuyo nivel de educación sea bajo, característica de gran parte de la encuesta de Monterrey. Los individuos que viven en sociedades en que el llenado de formas que solicitan información sobre historias vitales es frecuente adquieren cierta facilidad para recordar eventos discretos. Éste no es el caso de muchos de nuestros entrevistados porque viven en una tradición "oral". Esto no implica, sin embargo, que su capacidad para recordar hechos pasados sea necesariamente inferior a la de personas 
con mejor educación, pues a menudo recuerdan con detalle asombroso si se les da tiempo para reflexionar y oportunidad para encadenar sucesos como parte de una secuencia.

La mejor ayuda para recordar fue facilitada, según creemos, por la práctica de reunir información no sólo para un aspecto central, tal como la ocupación, sino para varios. La necesidad de pensar en diversos aspectos de la vida para un período dado de tiempo reforzó en lugar de debilitar la capacidad para recordar cada uno de dichos aspectos. Al relacionar distintos aspectos de su vida, el entrevistado fue capaz de dar razón más completa que de cualquier otra manera. ${ }^{15}$ Las respuestas resultaron también más dignas de crédito, ya que era el mismo entrevistado el que podía reconocer inconsistencias. Como cada aspecto tenía que ser congruente con los otros en cualquier lugar del tiempo, la posibilidad de que cualquiera de ellos se desfasara mucho respecto de los demás se redujo de esa manera. Al mismo tiempo, la tarea del encuestador se facilitó ya que, durante la entrevista, podía pedir aclaraciones o correcciones sobre inconsistencias $\mathbf{u}$ omisiones.

3. Control de la consistencia de las respuestas. El cuestionario fue diseñado de manera que la consistencia de las respuestas pudiera ser controlada. Se introdujo en otra parte del mismo un número de preguntas específicas que cubrian los mismos puntos que la historia vital; por ejemplo, preguntamos por el último empleo antes de migrar a Monterrey, el número y las edades de los hijos vivos, etc. Los encuestadores fueron instruidos para que durante la entrevista controlaran la consistencia de las dos fuentes cada vez que llegaran a una de esas preguntas. A su vez, el supervisor podía controlar la labor del encuestador en este aspecto.

En suma, creemos que los errores por falta de memoria fueron llevados al mínimo mediante los procedimientos que se acaban de describir. El énfasis en la secuencia y en la congruencia cronológica sirvió para reducir la probabilidad de olvidarse de registrar cambios de status o de colocar éstos fuera de secuencia. Probablemente la mayor fuente de error fue la de identificar el año específico de ocurrencia de los cambios. Pero es improbable que el fechado incorrecto involucrara más de unos pocos años ya que de otra manera la incongruencia con otras áreas se haría evidente.

La manera como se obtuvieron las historias vitales llevó a obtener tal vez mayor congruencia de la que pudo haber existido realmente. Se instruyó a los entrevistados para que insistieran cuando surgieran inconsistencias y probablemente algunos entrevistados hicieron sus historias más congruentes para "complacer" al encuestador. Sin duda hay también omisiones de cambios de status. No obstante, tales omisiones pueden ser menos graves de lo que parecen ya que con seguridad representan cambios de poca importancia en la vida del interrogado;

ฐ El lector puede probar esta afirmación por sí mismo. Debe, por ejemplo, tratar de recordar su lugar de residencia en un año específico (1954) o una edad específica (29) sin tomar en cuenta ningún otro aspecto de su vida. Le será difícil hacerlo sin pensar también en el lugar donde trabajaba, su situación familiar, etc. 
por ejemplo, puede haber cambiado de empleo con más frecuencia de la que notificó, pero los empleos omitidos fueron muy similares en carácter (Berelson y Steiner, 1964, p. 183).

El análisis de las historias vitales del estudio de Monterrey ha sido tranquilizador, ya que a la fecha no ha aparecido nada que no tenga sentido. Por supuesto que esto por sí solo no puede considerarse prueba de la confiabilidad de los datos, y como casi no hay manera de hacer verificaciones externas a las historias vitales, existirán siempre dudas respecto a tal información. Si se siguen cuidadosamente los procedimientos que se han descrito, pensamos que las distorsiones graves en las historias vitales quedarían reducidas a un mínimo.

\section{Elaboración de los datos}

Como ya se mencionó, aun en las escasas ocasiones en que se han recogido grandes cantidades de historias vitales, éstas no han sido analizadas generalmente en toda su extensión en vista de los formidables problemas involucrados en la elaboración de tan vasta cantidad de información (Taeuber, 1966). El uso extendido de las computadoras hace ahora factible la solución de este problema. En la investigación de Monterrey el primer paso del proceso fue desarrollar una técnica para transferir las historias vitales de los cuestionarios a las cintas magnéticas. Deseábamos conservar lo más posible de los datos de las historias vitales, pero al mismo tiempo reducir al mínimo el tiempo y el costo involucrados en la transferencia.

Dada la gran variación en longitud y contenido de las historias vitales según el entrevistado, los procedimientos usuales de codificación, que requieren "campos" comparables sobre los cuadros de codificación, tarjetas y cintas, no eran aplicables. El objetivo era pasar a las cintas las historias vitales completas, pero la codificación y la perforación de todos los datos habrían sido muy tardadas y costosas. Como teníamos treinta variables (cerca de la mitad con dos dígitos) y un máximo de 60 años, esto habría significado codificar aproximadamente 2700 campos por encuestado. Este procedimiento habría involucrado muchas repeticiones en las operaciones de codificación y perforación (por ejemplo, para un hombre de 50 años que siempre hubiera vivido en Monterrey el código que representara la residencia habría tenido que ser repetido 50 veces) y habría habido mucho espacio sin usar, especialmente en el caso de los entrevistados jóvenes.

Tratamos de salvar estas dificultades mediante la separación del proceso en dos conjuntos de operaciones: el primero, las operaciones de codificación y perforación, y el segundo la reconstrucción de la historia vital completa mediante la computadora. Sólo se codificaron y perforaron cambios en el contenido de cada variable. Cada cambio en el contenido de una variable fue codificado en un bloque de seis dígitos; los primeros dos identificaban la edad; el tercero y el cuarto la variable, y los últimos dos se referían al contenido específico de ésta. ${ }^{6}$ La codificación fue hecha en planillas grandes divididas en casi-

6 Hubo algunas variaciones a este acomodo. Dos variables conceptualmente 
llas, y cada casilla tenía ya impresas la edad y la identificación de la variable. En vista de que sólo se codificaron cambios en las variables, muchas casillas quedaron "incompletas".7 El anexo 2 representa una pequeña porción de una planilla de codificación.

La información fue luego transferida de las formas a las tarjetas, habiéndose perforado sólo las celdas con 6 dígitos. En el anexo 3 aparece la información codificada y perforada para las edades de 10 a 14 años de la historia vital ilustrativa (anexo 1). Como cada historia vital es distinta de las demás, no puede asignarse significado definido a algún campo particular de las tarjetas. ${ }^{8}$ El número de éstas réquerido para cada entrevistado varió con su edad y número de cambios registrados en cada una de las variables. El número promedio de tarjetas por entrevistado fue 3.4.

La codificación y perforación de la información fue un paso intermedio y resulta evidente que no se puede llevar a cabo ningún análisis directamente con las tarjetas. Fue preciso elaborar un programa especial para transferir la información de las tarjetas a las cintas, de manera que permitiera la reconstrucción de la historia vital completa a partir de las tarjetas con información sobre los cambios únicamente. Litzler escribió un programa general para este propósito. Este programa no sólo permite la reconstrucción de las secuencias referentes a las variables codificadas, sino que también permite la formación de nuevas variables y la condensación de otras. El programa Fortran lleva a cabo las siguientes cinco operaciones básicas en la reconstrucción de las historias de vida: 1) repite el valor de una variable cada año hasta encontrar un cambio en ella (por ejemplo, tamaño del lugar de residencia); 2) acumula el número de cambios de una variable dada (por ejemplo, número de lugares en que vivió el encuestado, registrado acumulativamente para cada año) ; 3) condensa las categorias de una variable dada en menos categorías (por ejemplo, un código industrial de 85 categorías queda reducido a uno de nueve) ; 4) crea variables "móviles" nuevas (el número de niños dependientes menores de 13 años, año por año); 5) transforma de acuerdo con instrucciones los valores de una variable dada (la transformación de los varios modos de ingreso declarado - diario, semanal, mensual, en pesos o dólares- a ingreso anual en pesos).

Creemos que un programa similar puede ser usado no sólo para historias vitales sino para cualquier tipo de información secuencial que involucre información de numerosas variables para muchas unidades de análisis. Tal programa podría ser usado en el análisis de las series de tiempo de un conjunto de variables de muchos países. También es útil en estudios longitudinales (desarrollo infantil, salud

separadas fueron combinadas bajo el mismo número de identificación siempre que ambas fueran variables de un dígito. Para una variable, el ingreso, sólo se usó un dígito para propósitos de identificación, de manera de dejar tres dígitos para el contenido.

7 Aun con la simplificación de los procedimientos de codificación, tomó aproximadamente 80 días-hombre completar e inspeccionar las 1640 historias vitales.

8 Todo lo que sabemos es que las columnas 7-8, 13-14, etc., se refieren siempre a la edad; las columnas 9-10, 15-16, etc., a la identificación de las variables, y las columnas 11-12, 17-18, etc., al contenido específico de las variables. 
y enfermedad, etc.) en los que solamente sea necesario registrar los cambios.

\section{ANÁLISIS}

El modo flexible de almacenar información en cintas magnéticas permite llevar a cabo numerosos análisis. En lo que sigue presentaremos primero algunos ejemplos de tipos de análisis que se han hecho a la fecha con los datos de Monterrey y luego elaboraremos acerca de otros campos de investigación en el que el presente enfoque pueda ser útil.

Debido a las limitaciones de espacio se describen sólo dos tipos de análisis de los que actualmente están en proceso. El primero es un análisis del avance ocupacional de los migrantes en Monterrey, tomando como variables independientes la edad y el año de llegada. Se trata de probar dos hipótesis relacionadas entre sí: 1) cuanto más joven sea el migrante, las probabilidades de avance ocupacional rápido en la ciudad son mayores; y 2) cuanto más temprana la fecha de llegada a Monterrey, mayor es el avance ocupacional, manteniendo constante el número de años vividos en Monterrey. Para probar estas hipótesis, los migrantes a Monterrey se clasifican por edad y año de llegada, y luego se comparan sus empleos antes de migrar con los empleos alcanzados en diferentes fechas después de su llegada. En seguida, es posible formar grupos pareados de migrantes y nativos y contrastar sus logros ocupacionales. Por ejemplo, el grupo de migrantes con edades entre 15 y 24 años de edad al llegar a Monterrey, llegados entre 1945 y 1949 se compara con el de nativos que tenían la misma edad en esos años. Tales comparaciones sirven para medir la ventaja relativa de nativos y migrantes en edades diferentes, en diferentes momentos en las historias y para un número especificado de años. Además de esto, pueden introducirse otras variables, tales como tamaño de la comunidad en que vivió el individuo antes de migrar a Monterrey o su estado civil en ese momento. La inclusión de otras variables no excluye conservar las variables de edad y año de llegada originales. La única limitación al análisis multivariado es, por supuesto, el tamaño de la muestra.

El segundo tipo de análisis por describir brevemente es la exploración de los determinantes y las consecuencias de cualquier suceso importante en el ciclo vital. Para ilustrar esto, podemos seleccionar como explanandum la edad al contraer matrimonio y luego tomar variables tales como educación, antecedentes familiares, tamaño de la comunidad en la que el individuo se crió, experiencia ocupacional previa, ocupación, y lugar de residencia en el momento del matrimonio, para determinar el valor explicativo que cada una de estas variables o varias de ellas en combinación pudieran tener. Más aún, se puede tomar la edad en el momento del matrimonio y ver sus efectos sobre características subsecuentes del individuo tales como el nivel de fecundidad y los logros ocupacionales. La edad a la que se obtuvo el primer empleo de tiempo completo es otro suceso del ciclo vital que está siendo analizado mediante un procedimiento similar.

A estas alturas debe estar claro que uno de los problemas princi- 
pales que todo investigador encuentra al trabajar con tales tipos de análisis es la falta de técnicas estadísticas apropiadas para manejar con carácter significativo los datos secuenciales. No obstante, en los últimos años los sociólogos y otros especialistas de las ciencias sociales han empezado a desarrollar tales técnicas y a dedicar más atención a los modelos causales. Los modelos estocásticos y los análisis de trayectoria son ejemplos de técnicas estadísticas adecuadas a los datos de historias vitales. ${ }^{9} \mathrm{El}$ aunar estos desarrollos metodológicos y estadísticos con los nuevos enfoques sobre la recolección y elaboración de datos de historias vitales en la forma descrita en este artículo debiera animar a más investigadores a diseñar sus investigaciones dentro de una configuración longitudinal.

El estudio de Monterrey se concentró principalmente en la movilidad ocupacional y geográfica, con cierta atención a la fecundidad; sin embargo, creemos que las técnicas desarrolladas para estos temas pueden ser usadas fácilmente en otros campos. Los estudios de comportamiento político, por ejemplo, suelen basarse en muestras nacionales en un momento del tiempo. En consecuencia, hay poco énfasis en la formación, mantenimiento y cambio del comportamiento y de las actitudes políticas. En este terreno tal vez los problemas de memoria serían mayores que para el caso de las historias residenciales y ocupacionales, pero si el voto o el comportamiento pueden ubicarse en el contexto de la vida del individuo los resultados pueden ser bastante satisfactorios. Este enfoque no sólo aumenta la confiabilidad de las respuestas, sino que permite al investigador buscar los determinantes y correlatos de las actitudes políticas.

La investigación en el área de las enfermedades mentales es otro ejemplo donde las ventajas de la historia vital no han sido convenientemente explotadas. Los psiquiatras y los psicólogos clínicos han desarrollado, por supuesto, técnicas para registrar las historias clínicas, pero rara vez se ha hecho un esfuerzo sistemático para incluir otras dimensiones tales como el empleo y la familia, junto con datos continuos sobre hospitalización y tratamiento psiquiátrico, en el estudio de muestras grandes. Tal enfoque puede llevar a interpretación más completa de los antecedentes y consecuencias de las enfermedades mentales. Los estudios de la relación entre las enfermedades mentales y la movilidad social y la migración, por ejemplo, han encontrado extremadamente difícil afirmar el valor de hipótesis alternativas referentes al orden temporal de las variables (Faris y Dunham, 1960; Dohrenwend, 1966). El hecho de que ciertos tipos de enfermedades mentales predominen en algunos estratos sociales, ¿es resultado de las condiciones sociales en ese estrato o de la selectividad social? Aunque hay un número de problemas técnicos involucrados en la prueba de estas hipótesis, la técnica de la historia vital

9 Los modelos estocásticos de movilidad ocupacional han sido empleados, por ejemplo, por Blumen et al. (1955) y Hodge (1966). En el campo de la migración interna, por Gurley (1965), Myers et al. (1967) y Morrison (1967). Este último estudio ha sido replicado con los datos de Monterrey por Land (1967). La técnica de los análisis de trayectoria ha atraído cada vez más la atención de los científicos sociales interesados en modelos causales (Blalock, 1964; Blau y Duncan, 1967; Duncan, 1966). 
puede ser útil, ya que es particularmente adecuada para contestar preguntas sobre la ordenación temporal de los sucesos en el ciclo vital.

\section{CONCLUSHONES}

Creemos que las historias vitales, registradas y manipuladas en la forma indicada, ofrecen mucho al investigador en ciencias sociales interesado en procesos así como en estructuras. No deseamos minimizar las dificultades de tal investigación, que requiere proyectos bien patrocinados, organizados de tal manera que incluyan encuestadores bien entrenados y bajo estrecha supervisión, codificadores expertos y acceso a computadoras de gran capacidad así como a programadores competentes. Nuestra experiencia en la encuesta de Monterrey nos ha convencido de que es posible recoger historias vitales confiables, y que la riqueza de los datos compensa con creces el esfuerzo requerido.

\section{BIBLIOGRAFIA}

Berelson, B. y Steiner, G. A., Human Behavior: An Inventory of Scientific Findings. Nueva York, Harcourt, Brace \& World, 1964.

Blalock, H. M., Causal Inferences in Non-Experimental Research. Chapel Hill, University of North Carolina Press, 1964.

Blau, P. M. y Duncan, O. D., The American Occupational Structure, Nueva York, John Wiley \& Sons, 1967.

Blumen, I., Kogan, M. y McCarthy, P. J., The Industrial Mobitity of Labor as a Probability Process (Cornell Studies in Industrial and Labor Relations, Núm. 6). Ithaca, Cornell University Press, 1955.

Bonilla, F. y Silva Michelena, J. A., Studying the Venezuetan Potity. Cambridge, M. I. T. Press, 1967.

Dohrenwend, B. P." "Social Status and Psychological Disorder: An Issue of Substance and An Issue of Method", American Sociological Review, 1966, Vol. 31, pp. 14-34.

Dollard, J., Criteria for the Life History. New Haven, Yale University Press, 1935.

Duncan, O. D., "Path Analysis: Sociological Examples", American Journal of Sociology, 1966, Vol. 72, pp. 1-16.

Faris, R. E. L. y Dunham, H. W., Mental Disorders in Urban Areas. Nueva York, Hafner, 1960.

Freedman, R., Whelpton, P. K. y Campbell, A. A., Family Ptanning, Sterility and Population Growth. Nueva York, McGraw-Hill, 1959.

Glass, D. V. (compilador), Social Mobility in Britain. Londres, Routledge \& Kegan Paul, 1954.

Gurley, W. R. "A Stochastic Analysis of Geographical Mobility and Population Projections of Census Divisions in the United States", Demography, 1965, Vol. 2, pp. 134-139.

Hodge, R. W., "Occupational Mobility as a Probability Process", Demography, 1966, Vol. 3, pp. 19-34.

Land, K., The Evaluation of a Stochastic Model of Migration: a Replication and Extension of Findings. Austin, Population Research Center, 1967, mimeografiado.

Lewis, O., The Children of Sánchez: Autobiography of a Mexican Family. Nueva York, Random House, 1961; Los hijos de Sánchez. México, J. Mortiz, 1964.

Lewis, O., La Vida: a Puerto Rican Family in the Culture of Poverty - San Juan and New York. Nueva York, Random House, 1966.

Morrison, P. A., "Duration of Residence and Prospective Migration: the Evaluation of a Stochastic Model", Demography, 1967, Vol. 4, pp. 553-561.

Myers, G. C., McGinnis, R. y Masnick, G., "The Duration of Residence Approach 
to a Dynamic Stochastic Model of Internal Migration: a Test of the Axiom of Cumulative Inertia", Eugenics Quarterly, 1967, Vol. 14, pp. 121-126.

Taeuber, K., Haenszel, W. y Sirken, M. G., "Residence Histories and Exposure Residences for the United States Population", Journal of the American Statistical Association, 1961, Vol. 56, pp. 824834.

Taeuber, K., "Cohort Migration", Demography, 1966, Vol. 3, pp. 416-422.

Wilensky, H. L., "Work, Careers, and Social Integration", International Social Science Journal, 1960 , Vol. 42, pp. 543-560.

Young, P. V., Scientific Social Surveys and Research. Englewood Cliffs, Prentice Hall, 1966. 
Anexo 1

ILUSTRACIÓN DE LA HISTORIA DB VIDA *

\begin{tabular}{|c|c|c|c|c|c|c|c|c|c|c|c|c|c|c|c|c|c|}
\hline \multirow{3}{*}{ za } & \multirow{3}{*}{$\begin{array}{l}m \\
\text { o } \\
0\end{array}$} & \multicolumn{4}{|c|}{ HISTORIA MIERATORIA } & \multirow{3}{*}{\multicolumn{2}{|c|}{ 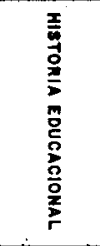 }} & \multirow{3}{*}{ 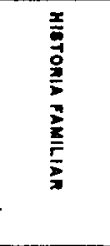 } & \multirow{3}{*}{ 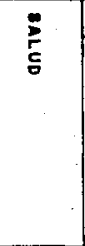 } & \multicolumn{7}{|c|}{ HISTORIA OCUPACIONAL } & \multirow{3}{*}{ 경 } \\
\hline & & \multirow[b]{2}{*}{ 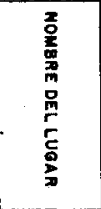 } & \multirow{2}{*}{$\begin{array}{l}\frac{m}{2} \\
\text { 咅 } \\
\text { 品 }\end{array}$} & \multicolumn{2}{|c|}{ TAMANTO } & & & & & : & 品 & 奠争 & EMPR & ESA & & $\overline{\mathbf{z}}$ & \\
\hline & & & & 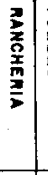 & & & & & & 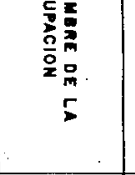 & 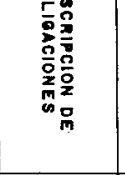 & 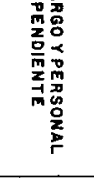 & 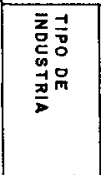 & 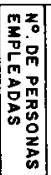 & 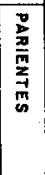 & 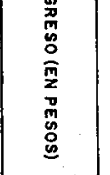 & \\
\hline 1940,1 & & San Isidro & coahuila & $x$ & & & $\begin{array}{l}\text { primaria } \\
\text { sol año } \\
\text { and }\end{array}$ & & & $\begin{array}{l}\text { trabojador } \\
\text { ogricolala }\end{array}$ & $\begin{array}{l}\text { ayuda a } \\
\text { su padre }\end{array}$ & 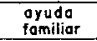 & agricuiftura & 3 & si & ninguno 1 & 1940 \\
\hline 4111 & i) & 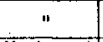 & $"$ & ". & & & 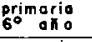 & & & $"$ & $n$ & " & ${ }^{\prime}$ & $"$ & $"$ & $"$ & 41 \\
\hline 421 & 21 & $\begin{array}{l}\text { Monterrey } \\
\text { (Col. Lomgs) }\end{array}$ & $\begin{array}{l}\text { Nuguo } \\
\text { Leon }\end{array}$ & & & 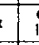 & $\begin{array}{l}\text { comercio } \\
\text { cer. oflo }\end{array}$ & & & $S ! N$ & EMPLEO & & & & & & 42 \\
\hline 431 & 3 & $"$ & 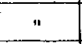 & & 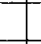 & 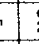 & $\begin{array}{l}\text { comerclo } \\
20 \\
20\end{array}$ & & & $\because$ & " & & & & & & 43 \\
\hline 44,1 & 4 & $"$ & " & & ( & 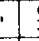 & 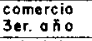 & & & " & 11 & & & & & & 44 \\
\hline 45, & 5 & Co.de Mexico & $\begin{array}{l}\text { Distrito } \\
\text { Federal }\end{array}$ & & 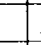 & 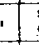 & 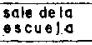 & & & mensajero & office boy & émpleádō- & $\begin{array}{l}\text { offeinade } \\
\text { F.F.C.C. } \\
\end{array}$ & 350 & no & $\begin{array}{c}300 \\
\text { mensuoles }\end{array}$ & 45 \\
\hline 46 1 & 16 & .1 & $n$ & & & & " & & & $"$ & " & $"$ & $"$ & $"$ & $n$ & $"$ & 46 \\
\hline $47+1$ & 17 & $"$ & " & & & & " & & & $"$ & " & $"$ & $"$ & $"$ &.$"$ & $"$ & 47 \\
\hline 481 & 18 & $\begin{array}{l}\text { Monterrey } \\
\text { (Col. Regino) }\end{array}$ & 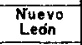 & & & & $"$ & & & SIN EMPLEO & POR 8 & MESES & & & 2 & & 48 \\
\hline 49 : & 9 & $"$ & $"$ & & & & $"$ & noviazgo & & oficinisto & $\begin{array}{c}\text { escribe cor tou } \\
\text { a moquina }\end{array}$ & $\begin{array}{c}\text { empleado } \\
0\end{array}$ & \begin{tabular}{|l|l|}
$\begin{array}{l}\text { fobricicode } \\
\text { colchones }\end{array}$ \\
\end{tabular} & 50 & $\operatorname{ma}$ & \begin{tabular}{|l|}
150 \\
semanales
\end{tabular} & 49 \\
\hline 19502 & 20 & $n$ & $"$ & & & 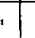 & $"$ & $"$ & & $"$ & 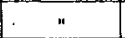 & $n$ & $\cdots$ & $\because$ & $\because$. & \begin{tabular}{|l|l|}
$n$ \\
\end{tabular} & 1950 \\
\hline 512 & 21111 & (Col. Modetor) & 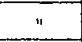 & & & & 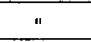 & matrimonio & & $"$ & $"$ & $"$ & $"$ & $"$ & $"$ & \begin{tabular}{|c|}
$18 O$ \\
semanales \\
\end{tabular} & 51 \\
\hline 52 & 22 & ." & " & & & 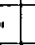 & $"$ & $\begin{array}{l}\text { nacimiento Ier. } \\
\text { hijo, hombre }\end{array}$ & & $\begin{array}{l}\text { agentie do } \\
\text { compras }\end{array}$ & $\begin{array}{l}\text { compro } 10000 \\
\text { los materialios }\end{array}$ & ${ }_{2}^{\text {empleado }}$ & $"$ & 70 & $"$ & \begin{tabular}{|c|}
250 \\
semianales \\
\end{tabular} & 52 \\
\hline 53 & 23 & “ & " & & & 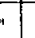 & $"$ & & & $n$ & $" 1$ & " & ". & $"$ & $"$ & n & 53 \\
\hline 54 & 24 & 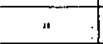 & " & & & $"$ & $"$ & 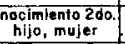 & & $\begin{array}{l}\text { propleforlade uno } \\
\text { pequent fobrical }\end{array}$ & gerente & pofrón & \begin{tabular}{|l|}
$\begin{array}{r}\text { rbricaco de } \\
\text { juguetes de }\end{array}$ \\
\end{tabular} & 6 & si & $\begin{array}{c}800 \\
\text { mensuales }\end{array}$ & 54 \\
\hline 55 & 25 & $"$ & $"$ & & & 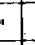 & $"$ & $\begin{array}{l}\text { nacimiento 3er- } \\
\text { hijo, hombre }\end{array}$ & $\begin{array}{l}\text { Murtic al } \\
\text { eer. mas }\end{array}$ & . & . & $"$ & \begin{tabular}{|l|l|} 
plosstico \\
\end{tabular} & $1 "$ & $"$ & " & 55 \\
\hline 56 & 26 & 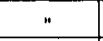 & $"$ & & & 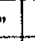 & $"$ & $\begin{array}{l}\text { nocimiento } 4^{\circ} \\
\text { hijo, hombre }\end{array}$ & & " & $"$ & $"$ & “ & 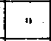 & $"$ & $"$ & 56 \\
\hline 572 & 27 & $"$ & $"$. & & & 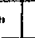 & $"$ & & & " & " & & $"$ & $"$ & " & 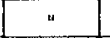 & 57 \\
\hline 58 & $2 \theta$ & $"$ & $"$ & & & 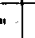 & $"$ & nocimionto $5^{\circ}$ & & n & $"$ & potrón & $"$ & 4 & " & 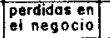 & 58 \\
\hline 59 & 29 & " & $"$ & & & $"$ & $"$ & & & $\begin{array}{l}\text { apentidide } \\
\text { ventes }\end{array}$ & visita tiendas & empleado & 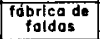 & 250 & no & 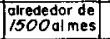 & 59 \\
\hline
\end{tabular}

* Sólo se presentan aquí 20 años de la vida del entrevistado. Para todos los entrevistados, el último año registrado fue el de la encuesta, 1965. 
Anexo 2

Sección ILUSTRATIVA DE LA HOJa DE CODIFICACIÓN

NUMERO DE VARIABLE
01
02
03
04
05

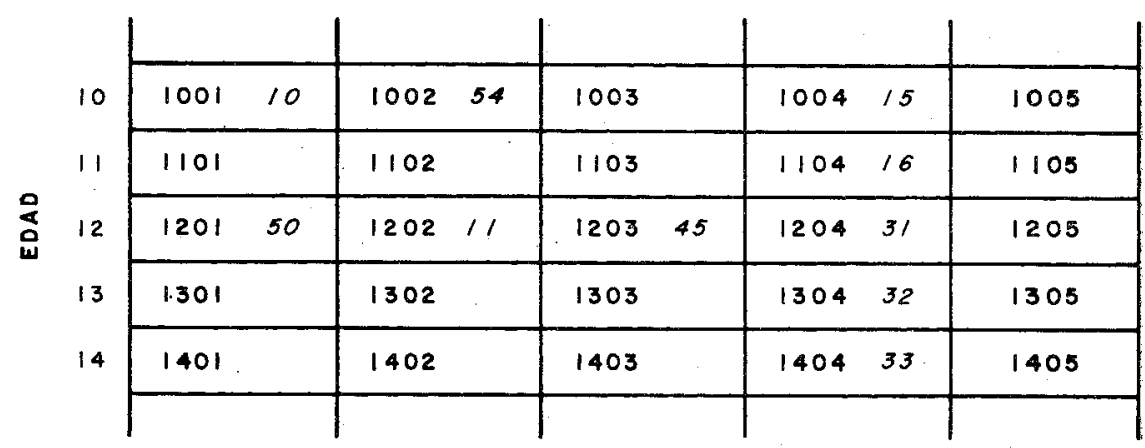

Anexo 3

Celdas dE SEIS dígitos QUE REgistran los CAMbios eN LAS EDADES 10 a 14 DE UNA "HISTORIa VITAL ILUSTRATIVA"

\begin{tabular}{|c|c|c|c|}
\hline Celda & Edad & Identificación de variable & Contenido de variable \\
\hline $10 \quad 01 \quad 10$ & 10 & Tamaño de la comunidad & Rural \\
\hline 100254 & 10 & Zona del país & Coahuila Norte \\
\hline 100415 & 10 & Educación & Primaria, 5: grado \\
\hline 100904 & 10 & Empleo & Trabaja todo el año \\
\hline 101051 & 10 & Posición & Ayuda a la familia \\
\hline 101101 & 10 & Actividad & Agricultura \\
\hline $1012 \quad 12$ & 10 & Parientes y número de empleados & Sí, de 2 a 5 \\
\hline 101401 & 10 & Uso de herramienta mecánica & Ninguna \\
\hline 101551 & 10 & Grupo ocupacional & Trabajador agrícola \\
\hline 104910 & 10 & Ingreso & Ninguno \\
\hline 110416 & 11 & Educación & Primaria, 6\% grado \\
\hline 120150 & 12 & Tamaño de la comunidad & Monterrey \\
\hline $12 \quad 0211$ & 12 & Zona del país & Monterrey \\
\hline 120345 & 12 & Colonia & Lomas \\
\hline 120431 & 12 & Educación & Comercio, 1er. año \\
\hline 120903 & 12 & Empleo & No trabaja, estudia \\
\hline 121099 & 12 & Posición & No aplicable \\
\hline 121199 & 12 & Actividad & No aplicable \\
\hline 121299 & 12 & Parientes y número de empleados & No aplicable \\
\hline 121499 & 12 & Uso de herramienta mecánica & No aplicable \\
\hline 121599 & 12 & Grupo ocupacional & No aplicable \\
\hline 124999 & 12 & Ingreso & No aplicable \\
\hline 130432 & 13 & Educación & Comercio, $2^{\circ}$ año \\
\hline 140433 & 14 & Educación & Comercio, 3er. año \\
\hline
\end{tabular}

